\title{
Intraocular Foreign Bodies-A Review from Entry to Exit and Beyond
}

\author{
Jiaxi Ding, MD, ${ }^{1}$ Sandra Fernando-Sieminski, MD² and Pradeepa Yoganathan, MD³
}

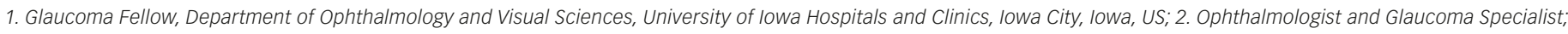
3. Ophthalmologist and Vitreoretinal Specialist, Department of Ophthalmology, Ross Eye Institute, The State University of New York, University at Buffalo, Buffalo, New York, uS

\begin{abstract}
Ophthalmic trauma, the leading cause of irreversible blindness in young adults, may be associated with obvious or occult intraocular foreign bodies (IOFBS). Critical work-up includes careful history-taking as well as slit-lamp and dilated eye examination. These components are frequently supplemented by imaging tests. Currently, computed tomography is the mainstream modality, offering highly sensitive detection and localization of IOFBS as small as $0.5 \mathrm{~mm}$. IOFBs impact visual prognosis by producing direct damage, causing secondary toxicity, and increasing infectious risk. Factors bearing a poor visual prognosis include large IOFB size, posterior segment location of IOFB, and retinal detachment or endophthalmitis on presentation. Timing of IOFB removal is controversial, but when possible, it is preferable to perform extraction within 24 to 48 hours of initial injury. Antibiotic coverage is also recommended with options of oral alone, intravenous followed by oral, or combination of intravitreal and oral. Even as technology and surgical techniques continue to improve IOFB management, we must still advocate accident prevention to our patients through education and emphasis of protective eyewear.
\end{abstract}

\section{Keywords}

Eye trauma, intraocular foreign body, traumatic endophthalmitis, chalcosis, siderosis

Disclosure: Jiaxi Ding, MD, Sandra Fernando-Sieminski, MD, and Pradeepa Yoganathan, MD, have no sources of support or financial disclosures to report. No funding was received in the publication of this article.

Open Access: This article is published under the Creative Commons Attribution Noncommercial License, which permits any noncommercial use, distribution, adaptation, and reproduction provided the original author(s) and source are given appropriate credit.

Received: May 1, 2015 Accepted: July 13, 2015 Citation: US Ophthalmic Review, 2015;8(2):135-8 DOl: http://doi.org/10.17925/USOR.2015.08.02.135

Correspondence: Jiaxi Ding, MD, Department of Ophthalmology and Visual Sciences, University of lowa Hospitals and Clinics, 200 Hawkins Drive, Iowa City, IA 52242 , US.

E: jiaxi84@gmail.com

In the US, approximately $3 \%$ of all emergency department visits are related to eye trauma. ${ }^{1}$ Many of these cases unfortunately lead to permanent visual impairment as ocular injury accounts for the leading cause of irreversible vision loss in teenagers and young adults. ${ }^{2,3}$ When penetrating or openglobe injuries occur, an estimated 10 to $41 \%$ involve an intraocular foreign body (IOFB). ${ }^{4-6}$ The IOFB may be overtly recognizable, but it may also be obscured by hemorrhage (as hyphema and/or in the vitreous), traumatic cataract, marked inflammation, or retinal detachment. At times, it could be inconspicuously lodged in the angle, in the sulcus, or in the far retinal periphery. Up to $20 \%$ of patients with IOFB present with no pain or decreased vision. 3,7 Therefore, a full and detailed ophthalmic examination including slit-lamp biomicroscopy and dilated examination is critical. Important information to be gained from tonometry, gonioscopy, ultrasonography, and scleral depression may not be feasible in the setting of globe penetration or perforation and if other concurrent systemic injuries warrant immediate medical attention. Overall, in the acute setting of eye trauma, the examiner must maintain a high index of suspicion for possible retained IOFBS.

\section{Mechanism of Injury}

The mechanism of injury can be a most important cue. Among the civilian population, the most common source of IOFB is work-related accidents with hammering being the largest culprit, followed by chiseling. ${ }^{8-10}$ In the military sector, trauma is frequently related to combat involving explosives with projectiles. These injuries resulting from blast fragments have been theorized to carry a lower infectious risk as the high speed and temperature may induce a sterilizing effect., $4,11,12$

\section{Epidemiology}

There is a clear preponderance in the demographics of patients presenting with IOFBs. More than $90 \%$ are young males with average age between 25 and 39 years. $3,4,10$ Most patients are relatively healthy with minimal co-morbidities and good visual acuity prior to the acute injury.

\section{Categorizations of Intraocular Foreign Bodies By Composition}

Broadly, IOFBs can be divided into metallic or nonmetallic subcategories, which can have implications upon modality of diagnosis, infectious risk, and Iong-term visual prognosis. Wichkham et al. reported that a vast majority (over $80 \%$ ) of IOFBs are metallic in nature.3,4,6,9 While metallic foreign bodies are associated with less risk for endophthalmitis compared with organic material, ${ }^{11}$ they may cause complications related to intraocular toxicity that can lead to acute and chronic visual loss, particularly with 
Figure 1: Thin-cut Sagittal and Axial Computed Tomography Views of a 35-year-old Male Presenting with Right Intraocular Foreign Body

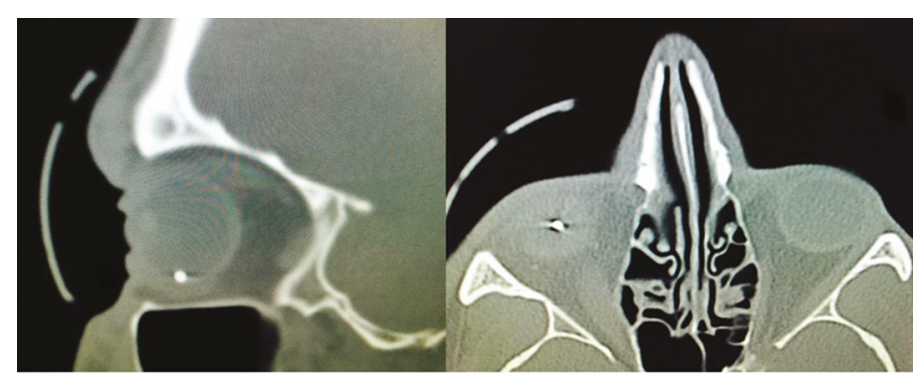

The patient was hammering steel while working on his automobile. On examination, he had hand motion vision, central stellate corneal laceration, and fluffy white lens material in the anterior chamber.

copper and iron IOFBS (see Toxicity sub-section in Complications and Sequelae). Common types of nonmetallic IOFBs include wood, stone, concrete, glass, and plastic. The latter two materials are considered inert and are generally well-tolerated in the eye, but nonmetallic organic IOFBS, such as wood, carry a higher endophthalmitis risk.11,13

\section{By Location}

IOFBS are usually located posteriorly, most commonly in the vitreous cavity followed by retinal or scleral embedding. ${ }^{4,6}$ For those IOFBs that are retained anteriorly (i.e., in the iris, angle, sulcus, anterior chamber, or lens), there is frequently a better prognosis for final visual acuity. ${ }^{6,14}$

\section{Diagnosis and Management}

A thorough history and full ophthalmic examination including dilated eye exam are the basic critical steps in cases of penetrating trauma and suspected IOFBs. When possible, gonioscopy and scleral depression should be considered at time of initial evaluation. There are several imaging options to facilitate diagnosing and locating IOFBS. Some studies have even described patterns of imaging subtleties to help deduce the IOFB composition. ${ }^{13}$ As technology and its availability has evolved, so has the test of choice. Each modality has its particular advantages and limitations.

\section{Imaging Modalities}

\section{Plain-film Radiographs}

Before the upsurge of computed tomography (CT) scans, plain-film radiographs were relied upon for identifying IOFBS. They are comparatively fast, inexpensive, easy to interpret, and have low exposure in regard to radiation dose. Unfortunately, they have limited sensitivity and only detect about $40 \%$ of IOFBS. ${ }^{15,16}$ Additionally, plain-films may produce up to $30 \%$ false negatives or inaccurate IOFB localization.9.17 Despite the overall movement away from reliance on $\mathrm{X}$-rays, in many instances, $\mathrm{X}$-rays are useful as a tool to screen for metallic foreign bodies before ordering magnetic resonance imaging (MRI). ${ }^{18}$

\section{Computed Tomography}

CT has become important in numerous diagnostic situations, particularly in the emergency department. It is now considered to be the mainstay of IOFB diagnosis. ${ }^{2}$ The majority of IOFBS are metallic ${ }^{3,4,9}-\mathrm{CT}$ imaging is the best modality for metallic IOFBs, detecting $100 \%$ of those greater than 0.5 to $0.6 \mathrm{~mm}$ with up to $100 \%$ accuracy. ${ }^{4,9,15,19-21}$ Clinically encountered
IOFBs range between 0.5 and $25 \mathrm{~mm}$ with mean size at $3.5 \mathrm{~mm} .^{13}$ Therefore, ophthalmologists often request thin axial cuts of the orbits at 0.5 to $1.0 \mathrm{~mm}$ for finer resolution (see Figure 1). For the IOFBs smaller than $0.5 \mathrm{~mm}$ in size and nonmetallic in nature (particularly glass, which may elude conventional $\mathrm{CT}$, helical $\mathrm{CT}$ has been shown to be more effective. ${ }^{4,8,22}$ Potential sources of false positives for metallic IOFB on CT include scleral bands, air bubbles, and dense intraocular hemorrhage. False negatives are likely due to movement during the scan process..$^{2,23}$

\section{Magnetic Resonance Imaging}

Compared with CTS, MRIs also have high detection sensitivity, show greater soft tissue resolution, and may be useful for detecting radiolucent IOFBs. ${ }^{15}$ However, they are rarely used for detecting IOFBs due to longer test duration, motion artifact, and most importantly frequent contraindication unless there is certainty as to the nonmetallic nature of the IOFB. ${ }^{13}$ Metallic fragments as small as $1 \mathrm{~mm}^{3}$ can be moved by the strong magnetic forces and damage surrounding tissues. ${ }^{2,4,18}$

\section{Ultrasonography}

Although extremely user-dependent, ultrasonography has a definite utility when appropriate. Ultrasound biomicroscopy can reveal IOFBs hidden by the iris or ciliary body. B-scan ultrasound can be up to $98 \%$ sensitive in detecting IOFBS and identify concurrent vitreous hemorrhage, retinal, or choroidal detachment. ${ }^{4,9}$ As previously mentioned, ultrasound should be deferred in circumstances involving penetrating injury to avoid risk for extruding intraocular contents due to direct pressure from the scanning probe or patient squeezing in discomfort.

\section{Medical and Surgical Considerations}

According to the US Eye Injury Registry, $3.4 \%$ of open-globe injuries involve endophthalmitis, ${ }^{11,24}$ which occurs in 3.1 to $30 \%$ of cases involving IOFBS. 4,11,12,15,20,25-36 Given the destructive nature of endophthalmitis, use of prophylactic antibiotic as soon as possible is generally practiced. The best treatment regimen for suspected traumatic endophthalmitis has not yet been determined, but frequent options include oral antibiotics alone, intravenous antibiotics followed by oral, or a combination of intravitreal and oral antibiotics. Vancomycin is the intravenous drug of choice, fluoroquinolones are most commonly used orally, and intravitreal formulation is usually with vancomycin (1 mg) and ceftazidime $(2.25 \mathrm{mg}){ }^{11}$

The patient's overall systemic well-being must be given first priority, and medical stability precedes consideration for eye surgery. Timing of IOFB removal is controversial, but when possible, it is preferable to perform extraction within 24 to 48 hours of initial injury. Additional factors for urgent ophthalmic surgical intervention may include concurrent eye complications, such as globe rupture, traumatic cataract, retinal detachment, and endophthalmitis on presentation. A basic summary of the management flow for IOFB is summarized in Figure 2.

\section{Complications and Sequelae}

As outlined in the review study by Bhagat et al., IOFBs impact visual prognosis in the following ways: (1) structural damage, (2) vehicle to deliver infectious agents, and (3) toxic reactions to the chemical nature of the IOFB. ${ }^{11}$ Larger size, trajectory, and final location of the IOFB are important factors in determining the extent of structural disruption (see section on Prognostic Factors Related to IOFB Injury). The most prominent sequelae related to 
retained IOFB in terms of infection, inflammation, and toxicity are further discussed below. Other complications of IOFB injuries include epiretinal membrane formation, glaucoma, and even choroidal neovascularization. 9,37

\section{Endophthalmitis}

When endophthalmitis is present, about half are identified pre-operatively and the other half postoperatively. ${ }^{4,38} \mathrm{An}$ increased risk is related to presence of retained IOFB, delay in wound closure beyond 24 hours, rural setting with soil contamination of the IOFB, and ruptured lens capsule. ${ }^{15,26,30,35}$ Compared with postsurgical endophthalmitis, traumatic endophthalmitis is more likely associated with virulent pathogens (such as Bacillus cereus, Staphylococcus, fungus, and polymicrobe) and therefore has a poorer prognosis. ${ }^{4}$ Combat-related IOFB injuries may carry a lower infectious risk as the high speed and temperature possibly help sterilize blast fragments. ${ }^{4,11,12}$

\section{Uveitis}

Noninfectious intraocular inflammation can be perpetuated by surgery or result from retained IOFB, phacoanaphylactic reaction, and sympathetic ophthalmia. ${ }^{11}$ The course of the inflammation can be fulminant and rapidly destructive. It can also be low-grade and chronic, manifesting inconspicuously as progressive cataract, transillumination iris defects, and recurrent hyphema or vitreous hemorrhage.3,4 Therefore, atypical and recurring unilateral uveitis in the setting of previous eye trauma should warrant consideration of occult retained IOFB. ${ }^{39}$

\section{Toxicity}

IOFBs of inert materials (such as glass, plastic, stone, and porcelain) are well-tolerated for long periods of time. Zinc and aluminum are minimally inflammatory, particularly if encapsulated. ${ }^{40}$ However, certain types of IOFB substances can elicit severe and destructive toxic chemical reactions within the eye. For example, foreign bodies with greater than $85 \%$ copper composition can generate marked inflammation with rapid irreversible deterioration of vision within hours. ${ }^{4,11,13,40}$ If the IOFB has a lower copper ion concentration, toxicity can manifest as a chronic mild chalcosis with signs including sunflower cataract, Kayser-Fleisher ring, and refractile crystals in the anterior chamber and retina. ${ }^{4}$

Intraocular iron is also regarded as highly toxic. In siderosis bulbi (see Figure 3), vision loss may be due to cataract, visual field loss, optic nerve atrophy, secondary open-angle glaucoma, and retina and retinal pigment

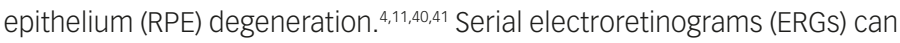
track the progression of siderotic retinal and RPE injury. In the early phase, b-wave amplitude decreases, and a-wave may be normal or abnormal. ${ }^{42}$ IOFB removal is recommended before amplitude reduction reaches $50 \%$ and becomes irreversible. Beyond this point, progression to ERG-signal extinction marks late-phase siderosis bulbi. 4,11,40-44

\section{Prognostic Factors Related to IOFB Injury}

There is general consensus that the following factors bear a worse visual prognosis in eyes with IOFB injury: large IOFB, posterior segment location of IOFB, and retinal detachment or endophthalmitis on presentation. $3,5,6,9,14,45,46$ The size and trajectory taken by the IOFB have important implications on visual prognosis. A larger IOFB is associated with more severe disruption of intraocular tissues. ${ }^{6}$ IOFBS with corneal entries typically inflict damage within the anterior segment given the lens barrier. This contends a
Figure 2: Intraocular Foreign Body Management Flow

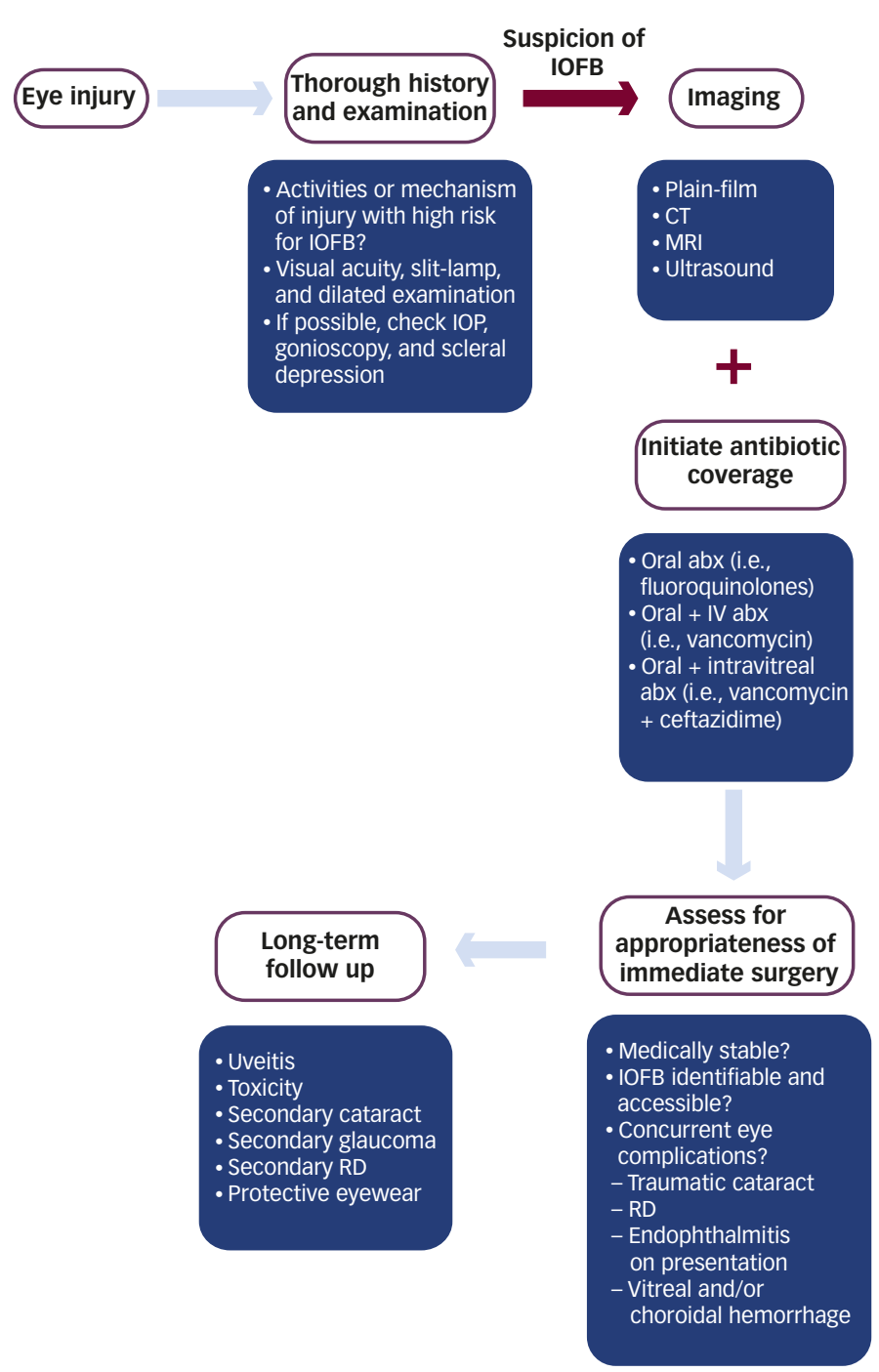

$A b x=$ antibiotics $; C T=$ computed tomography; IOFB = intraocular foreign body; IOP = intraocular pressure; $M R I=$ magnetic resonance imaging; $R D=$ retinal detachment .

Figure 3: Iris Heterochromia in a Patient with Old Retained Intraocular Foreign Body in the Left Eye and Subsequent Siderosis Bulbi

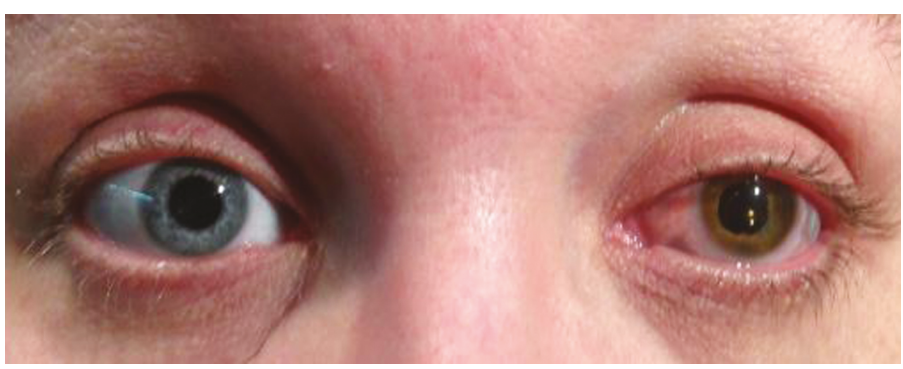

better prognosis than scleral or corneoscleral entry wounds that likely involve the posterior segment and higher risk for vitreous hemorrhage, endophthalmitis, retinal tears or detachment, and choroidal hemorrhage, rupture, or detachment. 4,6,14 
Other more-debated risk factors for poor ultimate prognosis include vision less than $20 / 200$ on presentation, time of IOFB removal, firearm injury, metallic composition, and use of silicone oil or external magnet at time of IOFB-removal surgery. $3,5,6,9$ The timing of IOFB removal is notably controversial. ${ }^{4}$ Some articles suggest that delayed IOFB removal beyond the initial 24 to 48 hours from injury increases risk for endophthalmitis. ${ }^{21,26,30,35,47,48}$ However, other reports indicate that early removal of IOFB may not be necessary. 10,12,14,49 The main contributors of the latter point come from military-related studies, which also describe that combat-sustained IOFBS may undergo sterilization due to high velocities and high temperatures from the explosive blast source before reaching the eye.

It is known that proliferative vitreoretinopathy (PVR) is major source of secondary failure in retinal detachment repair. PVR can be severe when stimulated by vitreous hemorrhage, early retinal tears with or without detachment, and delay of IOFB removal.5,9,11,12 Cardillo et al. reported 11.7 times risk for poor visual outcome in the presence of PVR. 9.50 Jonas et al. recommend that IOFBs be extracted within 24 hours to significantly lower rate of PVR formation. ${ }^{11,48}$

\section{Future Directions}

Great strides have been made in technology and surgical techniques that allow salvaging the globe and vision even in the face of devastating injuries. While we have yet to reach a standardized plan of action for IOFBS, acute treatment algorithms have been proposed.,3,8 Research, development, and refinement on the best management for IOFB trauma is and will be ongoing. Simultaneously, we must also educate about and emphasize the importance of injury prevention and protective eyewear to our patients, particularly those young and/or in industrial work environments.
1. Bord SP, Linden J, Trauma to the globe and orbit, Emerg Med Clin North Am, 2008;26:97-123, vi-vii.

2. Patel SN, Langer PD, Zarbin MA, Bhagat N, Diagnostic value of clinical examination and radiographic imaging in identification of intraocular foreign bodies in open globe injury, Eur J Ophthalmol, 2012;22:259-68.

3. Moisseiev E, Last $D$, Goez $D$, et al., Magnetic resonance imaging and computed tomography for the detection and characterization of nonmetallic intraocular foreign bodies, Retina, 2015;35:82-94.

4. Parke DW 3rd, Flynn HW Jr, Fisher YL, Management of intraocular foreign bodies: a clinical flight plan, Can $J$ Ophthalmol, 2013;48:8-12.

5. Erakgun T, Egrilmez S, Prognostic factors in vitrectomy for posterior segment intraocular foreign bodies, I Trauma 2008:64:1034-7.

6. Bai $H Q$, Yao L, Meng XX, et al., Visual outcome following intraocular foreign bodies: a retrospective review of 5-yea clinical experience, Eur J Ophthalmol, 2011;21:98-103.

7. Kuhn F, Halda T, Witherspoon CD, et al., Intraocular foreign bodies: myths and truths, Eur J Ophthalmol, 1996;6:464-71.

8. Yeh $\mathrm{S}$, Colyer $\mathrm{MH}$, Weichel ED, Current trends in the management of intraocular foreign bodies, Curr Opin Ophthalmol, 2008:19:225-33.

9. Wickham L, Xing W, Bunce C, Sullivan P, Outcomes of surgery for posterior segment intraocular foreign bodies - a retrospective review of 17 years of clinical experience, Graefes Arch Clin Exp Ophthalmol, 2006;244:1620-6.

10. Choovuthayakorn J, Hansapinyo L, Ittipunkul N, et al., Predictive factors and outcomes of posterior segment intraocular foreign bodies, Eye (Lond), 2011;25:1622-6.

11. Bhagat N, Nagori S, Zarbin M, Post-traumatic Infectious Endophthalmitis, Surv Ophthalmol, 2011:56:214-51.

12. Colyer MH, Weber ED, Weichel ED, et al., Delayed intraocular foreign body removal without endophthalmitis during Operations Iraqi Freedom and Enduring Freedom, Ophthalmology, 2007;114:1439-47

13. Modjtahedi BS, Rong A, Bobinski M, et al., Imaging characteristics of intraocular foreign bodies: a comparative study of plain film X-ray, computed tomography, ultrasound, and magnetic resonance imaging, Retina, 2015;35:95-104.

14. Chaudhry IA, Shamsi FA, Al-Harthi E, et al., Incidence and visual outcome of endophthalmitis associated with intraocular foreign bodies, Graefes Arch Clin Exp Ophthalmol, 2008;246:181-6

15. Ahmed Y, Schimel AM, Pathengay A, et al., Endophthalmitis following open-globe injuries, Eye (Lond), 2012:26:212-7.

16. Bryden FM, Pyott AA, Bailey M, MCGhee CN, Real time ultrasound in the assessment of intraocular foreign bodies, Eye (Lond), 1990;4(Pt 5):727-31.

17. Lit ES, Young LH, Anterior and posterior segment intraocular foreign bodies, Int Ophthalmol Clin, 2002;42:107-20.

18. Ta CN, Bowman RW, Hyphema caused by a metallic intraocular foreign body during magnetic resonance imaging, $\mathrm{Am} J$ Ophthalmol, 2000:129:533-4.

19. Chacko JG, Figueroa RE, Johnson $\mathrm{MH}$, et al., Detection and localization of steel intraocular foreign bodies using computed tomography. A comparison of helical and conventional axial scanning, Ophthalmology, 1997;104:319-23.

20. Maguire AM, Enger C, Eliott D, Zinreich SJ, Computerized tomography in the evaluation of penetrating ocular injuries, Retina, 1991:11:405-11.

21. Zhang Y, Zhang M, Jiang C, Qiu HY, Intraocular foreign bodies in China: clinical characteristics, prognostic factors, and visual outcomes in 1,421 eyes, Am J Ophthalmol, 2011:152:66-73.e1.

22. Gor DM, Kirsch CF, Leen J, et al., Radiologic differentiation of intraocular glass: evaluation of imaging techniques, glass types, size, and effect of intraocular hemorrhage, AJR AM J Roentgenol, 2001;177:1199-203.

23. Kubal WS, Imaging of orbital trauma, Radiographics, 2008;28:1729-39

24. Danis RP, Endophthalmitis, Ophthalmol Clin North Am, 2002:15:243-8.

25. Thompson JT, Parver LM, Enger CL, et al., Infectious endophthalmitis after penetrating injuries with retained intraocular foreign bodies, Ophthalmology, 1993;100:1468-74

26. Parke DW, Pathengay A, Flynn HW, et al., Risk factors for endophthalmitis and retinal detachment with retained intraocular foreign bodies, J Ophthalmol, 2012:2012:758526.

27. Andreoli CM, Andreoli MT, Kloek CE, et al., Low rate of endophthalmitis in a large series of open globe injuries, $A m$ Ophthalmol, 2009:147:601-8.

28. Affeldt JC, Flynn HW, Forster RK, et al., Microbial endophthalmitis resulting from ocular trauma, Ophthalmology, 1987;94:407-13.

29. Boldt HC, Pulido JS, Blodi CF, et al., Rural endophthalmitis, Ophthalmology, 1989;96:1722-6.

30. Snell Jr AC, Perforating ocular injuries, Am J Ophthalmol, 1945;28:263-81.

31. Edmund J, The prognosis of perforating eye injuries, Acta Ophthalmol, 1968;46:1165-74.

32. Niiranen M, Perforating eye injuries treated at Helsinsk University Eye Hospital 1970 to 1977, Ann Ophthalmol, 1981;13:957-61.

33. Barr CC, Prognostic factors in corneoscleral lacerations, Arch Ophthalmol, 1983;101919-24.
34. Essex RW, Yi Q, Charles PG, Allen PJ, Post-traumatic endophthalmitis, Ophthalmology, 2004;111:2015-22.

35. Yang CS, Lu CK, Lee FL, et al., Treatment and outcome of traumatic endophthalmitis in open globe injury with retained intraocular foreign body, Ophthalmologica, 2010;224:79-85.

36. Schrader WF, Epidemiology of open globe eve injuries: analysis of 1026 cases in 18 years, Klin Monatsbl Augenheilkd, 2004:221:629-35.

37. Fernández-López E, Desco-Esteban MC, Fandiño-López A, et al. Choroidal neovascularization after intraocular foreign body, Clin Ophthalmol, 2014;8:945-8.

38. Nashed A, Saikia P, Herrmann WA, et al., The outcome of early surgical repair with vitrectomy and silicone oil in openglobe injuries with retinal detachment, Am J ophthalmol, 2011:151:522-8.

39. Sychev $Y$, Verner-Cole EA, Suhler EB, et al., Occult nonmetallic intraocular foreign bodies presenting as fulminant uveitis: a case series and review of the literature, Clin Ophthalmol, 2013;7:1747-51.

40. Luo Z, Gardiner M, The incidence of intraocular foreign bodies and other intraocular findings in patients with corneal metal foreign bodies, Ophthalmology, 2010;117:2218-21.

41. Xie H, Chen S, Ocular siderosis, Eye SCi, 2013;28:108-12.

42. Schechner R, Miller B, Merksamer E, Periman I, A long term follow up of ocular siderosis: quantitative assessment of the electroretinogram, Doc Ophthalmol, 1990-1991;76:231-40.

43. Weiss MJ, Hofeldt AJ, Behrens M, Fisher K, Ocular siderosis Diagnosis and management, Retina, 1997;17:105-8.

44. Knave B, Eletroretinography in eyes with retained intraocular metallic foreign bodies, Acta Ophthalmol, 1969;100(Suppl.):5-63.

45. Wani VB, Al-Aimi M, Thalib L, et al., Vitrectomy for posterior segment intraocular foreign bodies: visual results andprognostic factors, Retina, 2003:23:654-60.

46. Woodcock MG, Scott RA, Huntbach J, Kirkby GR, Mass and shape as factors in intraocular foreign body injuries, Ophthalmology, 2006;113:2262-9.

47. Chaudhryl A, Shamsif A, Al-Harthi E, et al., Incidence and visual outcome of endophthalmitis associated with intraocular foreig bodies, Graefes Arch Clin Exp Ophthalmol, 2008;246:181-6.

48. Jonas $J$, Knorr $\mathrm{H}$, Budde W, Prognostic factors in ocular injuries caused by intraocular or retrobulbar foreign bodies, Ophthalmology, 2000;107:823-8.

49. Thach A, Ward T, Dick JN, et al., Intraocular foreign body injuries during Operation Iraqi Freedom, Ophthalmology 2005;112:1829-33

50. Cardillo JA, Stout JT, LaBree L, et al., Post-traumatic proliferative vitreoretinopathy. The epidemiologic profile, onset, risk factors and visual outcome, Ophthalmology, 1997;104:1166-73. 